\title{
Inkjet-Printed Flexible Temperature Sensor Based on Silver Nanoparticles Ink ${ }^{\dagger}$
}

\author{
Qiao Jun Liew ${ }^{1,2}$, Aiman Sajidah Abd Aziz ${ }^{1}$, Hing Wah Lee ${ }^{1}$, Mai Woon Lee ${ }^{1}$, Huzein Fahmi \\ Hawari ${ }^{2}$ and Mohd Haris Md Khir ${ }^{2, *}$ \\ 1 Flexible Electronics Laboratory, MIMOS Berhad, Kuala Lumpur 57000, Malaysia; qj.liew@mimos.my \\ (Q.J.L.); aiman.aziz@mimos.my (A.S.A.A.); hingwah.lee@mimos.my (H.W.L.); mw.lee@mimos.my (M.W.L.) \\ 2 Department of Electrical and Electronic Engineering, Universiti Teknologi PETRONAS, Seri Iskandar \\ 32610, Perak, Malaysia; huzeinfahmi.hawari@utp.edu.my \\ * Correspondence: hingwah.lee@mimos.my \\ + Presented at the 7th Electronic Conference on Sensors and Applications, 15-30 November 2020; Available \\ online: https://ecsa-7.sciforum.net/.
}

Published: 15 November 2020

\begin{abstract}
In this research, a flexible inkjet-printed temperature sensor with in-house silver nanoparticles ink is presented and compared with the sensor printed with commercial silver nanoparticles ink. These sensors have an average width of $0.5 \pm 0.04 \mathrm{~mm}$ in the latter and $0.5 \pm 0.03$ $\mathrm{mm}$ in the former. These serpentine structure sensors was printed on polyethylene terephthalate (PET) substrate by using Fujifilm Dimatix 2850 printer. The corresponding results indicated in resistance have been recorded in the range of $30-100{ }^{\circ} \mathrm{C}$ to evaluate the sensor performance. The result of the studies showed that there was a linear relationship between the resistance and temperature for both ink types. The printed sensors developed using the in-house ink presented higher sensitivity $0.1086 \Omega /{ }^{\circ} \mathrm{C}$ compared to the commercial ink which was $0.0543 \Omega /{ }^{\circ} \mathrm{C}$. Therefore, the flexible inkjet-printed temperature sensor with the in-house silver nanoparticles ink is recommended for the large-scale productions and implementations.
\end{abstract}

Keywords: Inkjet-printed; flexible substrate; temperature sensor; silver nanoparticles; printed electronics

\section{Introduction}

The emerging of IoT has radically caused the printed electronics and fabrication technologies to advance since the printing techniques have been industrialized. Today, paper-based electronics in the fields of flexible devices and wearables has gained great interests to be utilized in wide area electronic systems because of its low-cost in setup and methodology, as well as its eco-friendly fabrication technologies. Therefore, its development has become essentially significant to co-exist with conventional silicon-based manufacturing systems to be deployed in IoT devices.

Among of the IoT technologies, the temperature sensor is one of the most widely employed in many field such as wearable sensor and logistics monitoring system [1,2]. Most of the publications reported the temperature sensors as the resistive temperature detectors [3]. In order to fabricate high performance temperature sensor, the type and quality of the ink is one of the challenge of the printed electronics industry. For instance, researches in [4] and [5] have claimed their device performances are excellent by using gold $(\mathrm{Au})$ and copper $(\mathrm{Cu})$ inks respectively. However, the temperature of the paper substrate is not able to withstand both the required curing temperatures of the Au and $\mathrm{Cu}$ inks. Compared to the $\mathrm{Au}$ and $\mathrm{Cu}$ inks, the silver nanoparticle ink (AgNP) has been highly recommended due to AgNP has low reactivity to air [6], minimal resistance to corrosion [7], lower melting temperature [8] and lower cost [9]. Because of these, the commercial silver ink has dominating the 
market. However, the cost has become the one of the greatest concern to the industrial interest as the cost of large-scale productions is high.

In this research, the low-cost in-house and flexible inkjet-printed temperature sensor with inhouse silver nanoparticles ink is studied and compared to the sensor printed with commercial silver nanoparticles ink. The outcome of the research has shown that the performance of the temperature sensing from the in-house sensor is very comparable to the commercial sensor.

\section{Materials and Methods}

\subsection{Temperature Sensor Design}

In thispaper, a meander shape type temperature sensor is designed with the dimension of 6.9 $\mathrm{mm} \times 13.2 \mathrm{~mm}$. The width of the sensor is $0.5 \mathrm{~mm}$ and gap is $0.4 \mathrm{~mm}$. The padding area is $1.5 \mathrm{~mm} \times$ $1.5 \mathrm{~mm}$. Figure 1 shows the design of a temperature sensor.

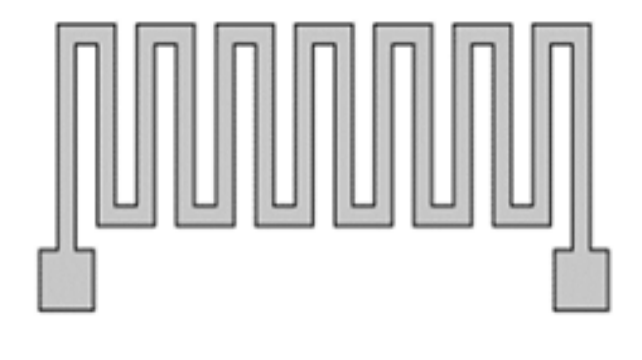

Figure 1. Temperature sensor design

\subsection{Flexible Substrate and Ink Preparation}

The PET substrate used in this study was purchased from Elephantech Inc. with a thickness of $107 \mu \mathrm{m} \pm 32 \mu \mathrm{m}$. The PET comes in A4 size and can be cut into the desired size based on the application. This PET substrate has been treated by the manufacturer and printed with the design directly.

In this study, there are two types of silver nanoparticles ink used to print the temperature sensor. First one is the commercial silver nanoparticles ink purchased from Novacentrix (JS-B25HV). It is designed to print devices/circuits on coated substrate including PET. Besides that, this ink is designed for the Dimatix Fujifilm printer and the printing waveform is provided.

Another type for silver nanoparticles ink (AgNPs ink) used in this study is developed by our researcher. The idea of developing the in-house silver nanoparticles is to produce a low-cost silver nanoparticles ink and avoid the post-printing process (thermal cure). Table 2 shows the physical properties of in-house Silver nanoparticles ink.

Table 1. Physical properties for JS-B25HV and in-house AgNPs ink.

\begin{tabular}{ccc}
\hline Properties & JS-B25HV & In-House AgNPs Ink \\
\hline Ag content $\left(\mathrm{wt}^{\mathrm{m}} \mathrm{)}\right)$ & 25 & 10 \\
\hline Viscosity $(\mathrm{cP})$ & $8-10$ & 6.5 \\
\hline Surface tension $($ dyne $/ \mathrm{cm})$ & $30-32$ & $57-66$ \\
\hline Particle size $(\mathrm{nm})$ & $60-80$ & $30-60$ \\
\hline
\end{tabular}

\subsection{Inkjet Printing}

The temperature sensor fabricated by inkjet printing technique using Fujifilm Dimatix printer (DMP 2850). The DMP printer offers drop-on demand-demand (DOD) piezoelectric inkjet technology which allows to deposit fluids materials on substrate. It allows users to modify the printer parameters in order to obtain the highest printing quality of products based on their fluids/inks. The Silver nanoparticle ink is required to be sonicated around $15 \mathrm{~min}$ to avoid the agglomerate between the 
particles. The fluid $(\sim 3 \mathrm{~mL})$ is then put into the cartridge with a syringe, a needle and 0.2 um filter. The filter is used to filter out the particle that bigger than $0.2 \mathrm{um}$ to prevent the nozzle head clog. It is recommended to keep the cartridge at least $30 \mathrm{~min}$ in idle state after filling.

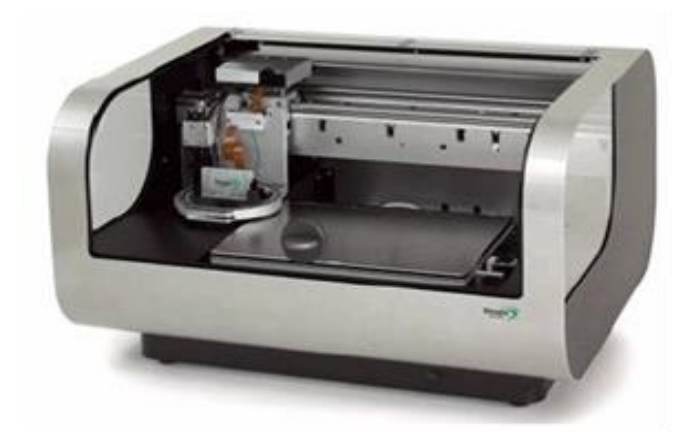

Figure 2. DMP-2850 inket printer.

The IDEs designs are established by using COMSOL Multiphysics and exported as CAD data file (.dxf). ACE 3000 converter software is used to convert the CAD data file into bitmap file (.bmp). The drop spacing is chosen before exporting it in bitmap file. Finally, the bitmap file is loaded in DMP program. The bitmap file is saved as the pattern file (.ptn) using the Pattern Editor (Bitmap images) on DMP program. The number layer, image resolution and the leader bar are set properly according to design specifications. It is noted that the image resolution must match to the drop spacing setting to avoid dimension run out of design specifications.

In order to find the optimum deposition recipe, printer parameters need to be adjust based on the experimental outcome by trial-and-error. The printer parameters that being investigated: substrate thickness, tickle control, plate/stage temperature, cartridge temperature, jetting voltage, meniscus setpoint, number of jets to use, and cartridge print height

Table 3 shows the optimal printing parameters that are used for commercial ink (JS-B25HV) while Table 4 shows the optimal printing parameters used for the in-house ink.

Table 2. Printing parameter for JS-B25HV.

\begin{tabular}{cc}
\hline Printer Parameter & Value \\
\hline Substrate Thickness $(\mathrm{um})$ & 200 \\
\hline Tickle Control $(\mathrm{kHz})$ & $5 \mathrm{kHz}$ \\
\hline Plate Temperature $\left({ }^{\circ} \mathrm{C}\right)$ & 40 \\
\hline Cartridge temperature $\left({ }^{\circ} \mathrm{C}\right)$ & 30 \\
\hline Jetting Voltage $(\mathrm{v})$ & 22 \\
\hline Meniscus Setpoint $\left(\right.$ inches $\left.\mathrm{H}_{2} \mathrm{O}\right)$ & 3.5 \\
\hline Cartridge Print Height $(\mathrm{mm})$ & 0.5 \\
\hline Number of jets to use & 1 \\
\hline
\end{tabular}

Table 3. Printing parameter for in-house ink.

\begin{tabular}{cc}
\hline Printer Parameter & Value \\
\hline Substrate Thickness $(\mathrm{um})$ & 200 \\
\hline Tickle Control $(\mathrm{kHz})$ & $5 \mathrm{kHz}$ \\
\hline Plate Temperature $\left({ }^{\circ} \mathrm{C}\right)$ & 40 \\
\hline Cartridge temperature $\left({ }^{\circ} \mathrm{C}\right)$ & 30 \\
\hline Jetting Voltage $(\mathrm{v})$ & 15 \\
\hline Meniscus Setpoint $\left(\right.$ inches $\left.\mathrm{H}_{2} \mathrm{O}\right)$ & 4 \\
\hline Cartridge Print Height $(\mathrm{mm})$ & 0.5 \\
\hline Number of jets to use & 1 \\
\hline
\end{tabular}




\subsection{Sensor Characterization}

The geometrical characterization is performed using optical microscope. Microscope is used to make sure the printed sensors matched to the design and to monitor printed quality visually.

The temperature sensor is characterized by measuring the resistance between $30^{\circ} \mathrm{C}$ and $100{ }^{\circ} \mathrm{C}$ with increment of $10^{\circ} \mathrm{C}$. The resistance values is measured using the FLUKE digital multi-meter.

\section{Results and Discussion}

\subsection{Geometrical Characterization}

Before the electrical characterization, the printed sensors have been observed using Olympus microscope to investigate the surface morphology and uniformity. Figures 3 and 4 show the microscope image for both ink types. Besides that, the dimension of printed sensors is measured and compared to the design layout. Table 5 show the comparison between the design layout and the actual printed. The inkjet printing methods present several adverse effects that cause the inaccuracy on the printed structures. Based on the microscope result, the percentage errors for all the printed sensors are less than $10 \%$ which is considered as the acceptable results.
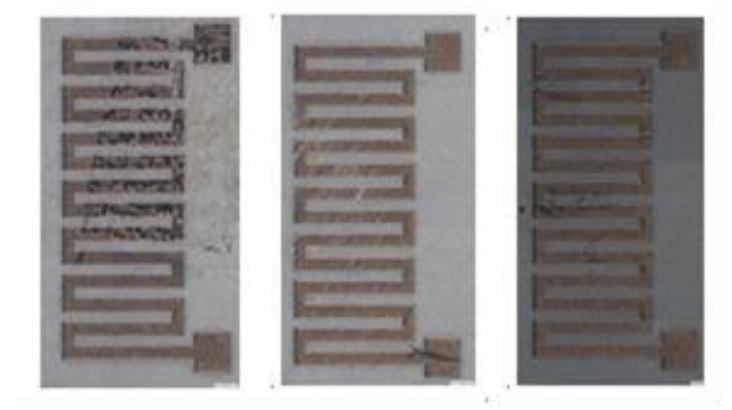

Figure 3. Microscope image for JS-B25HV.

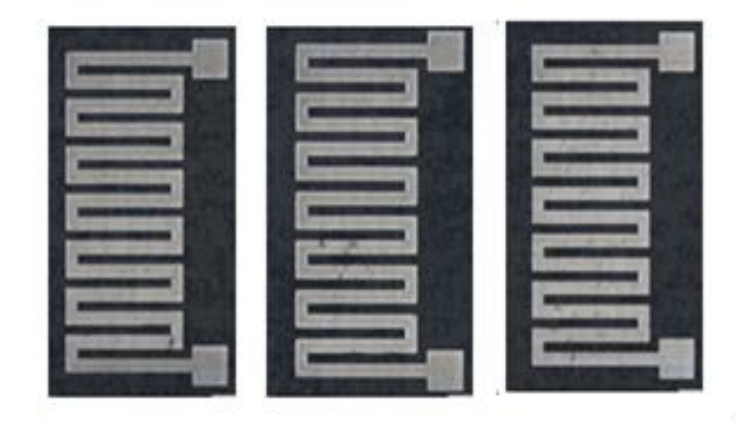

Figure 4. Microscope image for in-house ink.

Table 4. Comparison the sensor dimension between design layout and actual printed sensor.

\begin{tabular}{cccc}
\hline Parameter & Design & JS-B25HV & In-house Ink \\
\hline $\begin{array}{c}\text { Finger width }(\mathrm{mm}) \\
\begin{array}{c}\text { Gap between fingers } \\
(\mathrm{mm})\end{array}\end{array}$ & 0.5 & $0.5 \pm 0.04$ & $0.5 \pm 0.03$ \\
\hline
\end{tabular}




\subsection{Electrical Characterazation}

The performance of temperature sensors are evaluated in the temperature range of $30{ }^{\circ} \mathrm{C}$ to 100 ${ }^{\circ} \mathrm{C}$ with increment of $10{ }^{\circ} \mathrm{C}$ each time step. Figure 5 show the relationship between resistance and temperature both ink types. There are total of six experiments that have been carried out. All of these show linear relationship between resistance and temperature.
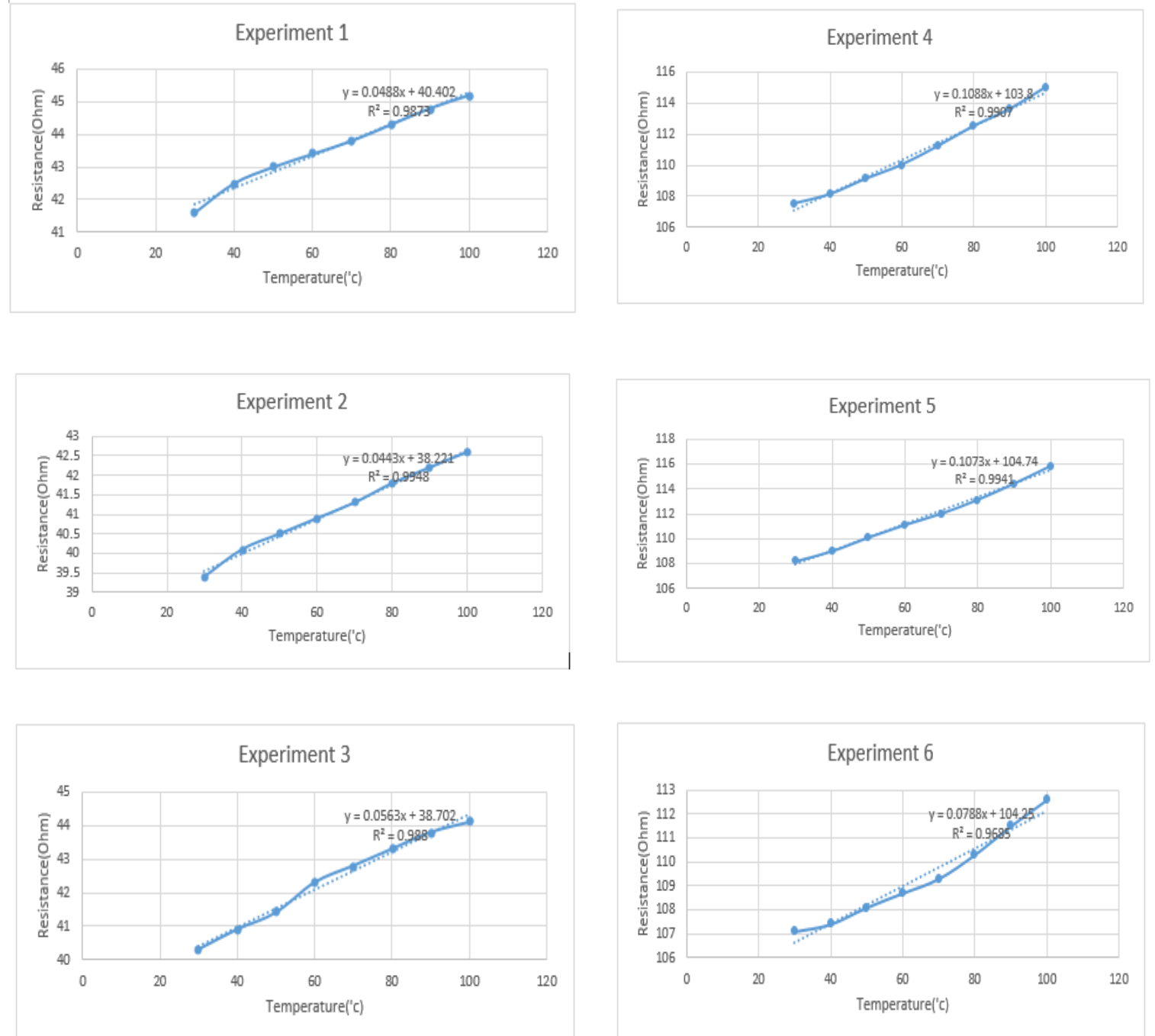

Figure 5. Relationship between resistance and temperature for JS-B25HV (Experiement1-3); for Inhouse ink(Experiment 4-6).

The Temperature Coefficient of Resistance (TCR) can be calculated by

$$
T C R=\frac{R_{100}-R_{30}}{R_{30}(\Delta T)}
$$

where $R_{100}$ and $R_{30}$ denote the resistance value obtained at temperature $100{ }^{\circ} \mathrm{C}$ and $30{ }^{\circ} \mathrm{C}$ respectively, $\Delta \mathrm{T}$ is the change in temperature. The definition of TCR is the resistance change factor per degree Celsius of temperature change.

Besides that, the sensitivity $(S)$ of the temperature sensors can be calculated by

$$
S=\frac{R_{100}-R_{30}}{\Delta T}
$$


where $R_{100}$ and $R_{30}$ denote the resistance value obtained at temperature $100{ }^{\circ} \mathrm{C}$ and $30{ }^{\circ} \mathrm{C}$ respectively, $\Delta T$ is the change in temperature. The result in Table 6 show that the printed temperature sensor that fabricated using in-house ink obtained high sensitivity performance compared to the commercial ink.

Table 6. Sensitivity and TCR of printed temperature sensor.

\begin{tabular}{ccc}
\hline Experiment & Temperature Coefficient of Resistance $\left(\mathrm{c}^{-1}\right)$ & Sensitivity $\left(\Omega /{ }^{\circ} \mathrm{C}\right)$ \\
\hline 1 & $12.3626 \times 10^{-4}$ & 0.0514 \\
\hline 2 & $11.6026 \times 10^{-4}$ & 0.0457 \\
\hline 3 & $13.4704 \times 10^{-4}$ & 0.0543 \\
\hline 4 & $9.9668 \times 10^{-4}$ & 0.1071 \\
\hline 5 & $10.0343 \times 10^{-4}$ & 0.1086 \\
\hline 6 & $7.3363 \times 10^{-4}$ & 0.0786 \\
\hline
\end{tabular}

\section{Conclusions}

The flexible temperature sensors were developed through inkjet printer (DMP2850) using two type of ink: JS-B25HV and in-house Silver nanoparticles ink. The performance of the printed sensors were evaluated by vary the temperature from $30^{\circ} \mathrm{C}$ and $100{ }^{\circ} \mathrm{C}$. The result shows that the printed sensors have linear relationship between resistance and temperature for both ink. The printed sensors developed using in-house ink give the higher sensitivity which is $0.1086 \Omega /{ }^{\circ} \mathrm{C}$ compared to the commercial ink. Therefore, the in-house ink is comparable to the commercial ink and has great potential for industrial applicability.

Author Contributions: Conceptualization, Q.J.L., H.F.H. and H.W.L.; methodology, Q.J.L. and A.S.A.A.; validation, Q.J.L., A.S.A.A. and M.W.L.; writing-original draft preparation, Q.J.L.; writing-review and editing, H.W.L.; visualization, Q.J.L.; supervision, H.W.L., H.F.H. and M.H.M.K.; funding acquisition, H.W.L. All authors have read and agreed to the published version of the manuscript.

Funding: This research was funded by ministry of Science, Technology and Innovation (MOSTI), grant number P0110020110122.

Acknowledgments: We would like to acknowledge the financial support provided by ministry of Science, Technology and Innovation (MOSTI) through the 11th Malaysia Plan development expenditure (DE) funding.

Conflicts of Interest: The authors declare no conflict of interest

\section{References}

1. Barmpakos, D.; Segkos, A.; Tsamis, C.; Kaltsas, G. A Disposable Inkjet-Printed Humidity and Temperature Sensor Fabricated on Paper. Proceedings 2018, 2, 977.

2. Ali, S.; Khan, S.; Bermak, A. Inkjet-Printed Human Body Temperature Sensor for Wearable Electronics. IEEE Access 2019, 7, 163981-163987, doi:10.1109/access.2019.2949335.

3. Dankoco, M.D.; Tesfay, G.Y.; Benevent, E.; Bendahan, M. Temperature sensor realized by inkjet printing process on flexible substrate. Mater. Sci. Eng. B 2016, 205, 1-5, doi:10.1016/j.mseb.2015.11.003.

4. Cui, W.; Lu, W.; Zhang, Y.; Lin, G.; Wei, T.; Jiang, L. Gold nanoparticle ink suitable for electric-conductive pattern fabrication using in ink-jet printing technology. Colloids Surfaces A: Physicochem. Eng. Asp. 2010, 358, 35-41, doi:10.1016/j.colsurfa.2010.01.023.

5. Lim, S.; Joyce, M.; Fleming, P.D.; Aijazi, A.T.; Atashbar, M. Inkjet Printing and Sintering of Nano-Copper Ink. J. Imaging Sci. Technol. 2013, 57, 1-7, doi:10.2352/j.imagingsci.technol.2013.57.5.050506.

6. Öhlund, T.; Schuppert, A.; Andres, B.; Forsberg, S.; Andersson, H.; Schmidt, W.; Nilsson, H.-E.; Zhang, R.; Olin, H. Assisted sintering of silver nanoparticle inkjet ink on paper with active coatings. RSC Adv. 2015, 5, 64841-64849, doi:10.1039/C5RA06626C.

7. Wignes, F.L.; Glascoe, C.; Makar, G.; Karacolak, T.; Sekhar, P. A paper based 2-element antenna for WLAN MIMO applications fabricated using inkjet technology. Microw. Opt. Technol. Lett. 2017, 59, 2785-2790, doi:10.1002/mop.30822. 
8. Hassan, A.; Ali, S.; Hassan, G.; Bae, J.; Lee, C.H. Inkjet-printed antenna on thin PET substrate for dual band Wi-Fi communications. Microsyst. Technol. 2016, 23, 3701-3709, doi:10.1007/s00542-016-3113-y.

9. Huang, Q.; Shen, W.; Fang, X.; Chen, G.; Guo, J.; Xu, W.; Tan, R.; Song, W. Highly flexible and transparent film heaters based on polyimide films embedded with silver nanowires. RSC Adv. 2015, 5, 45836-45842, doi:10.1039/c5ra06529a.

10. Saha, T.K.; Knaus, T.N.; Khosla, A. Investigation of Printing Properties on Paper Substrate. J. Electrochem. Soc. 2018, 165, B3163.

Publisher's Note: MDPI stays neutral with regard to jurisdictional claims in published maps and institutional affiliations.

(C) 2020 by the authors. Submitted for possible open access publication under the terms and conditions of the Creative Commons Attribution (CC BY) license (http://creativecommons.org/licenses/by/4.0/). 